Proc. 15th Int. Conference on Defects Recognition, Imaging and Physics in Semiconductors, Warsaw, Poland 2013

\title{
Effect of Deep-Level Defects on Transient Photoconductivity of Semi-Insulating $4 \mathrm{H}-\mathrm{SiC}$
}

\author{
M. Suproniuk ${ }^{a, b, *}$, P. KAmiński ${ }^{c}$, R. KozŁowski ${ }^{c}$ And M. PAwŁowski ${ }^{a}$ \\ ${ }^{a}$ Military University of Technology, S. Kaliskiego 2, 00-908 Warszawa, Poland \\ ${ }^{b}$ Maria Skłodowska-Curie Warsaw Academy, Łabiszyńska 25, 03-204 Warszawa, Poland \\ ${ }^{c}$ Institute of Electronic Materials Technology, Wólczyńska 133, 01-919 Warszawa, Poland
}

\begin{abstract}
A model enabling the equilibrium conductivity and transient photoconductivity of semi-insulating $4 \mathrm{H}-\mathrm{SiC}$ to be simulated has been demonstrated. Using this model, the simulations of both equilibrium conductivity and transient photoconductivity have been carried out. Both the simulation and experimental results have shown that the evolution of photoconductivity in time after switching on the band-to-band generation of electron-hole pairs is strongly affected by the properties of deep level defects. The results of transient photocurrent measurements confirm the simulations results indicating that the $Z_{1 / 2}$ center is a very effective recombination center in semi-insulating $4 \mathrm{H}-\mathrm{SiC}$ having detrimental effect on the transient photoconductivity.
\end{abstract}

DOI: $10.12693 /$ APhysPolA.125.1042

PACS: 72.40.+w, 71.55.-i, 72.20.-i

\section{Introduction}

Transient photoconductivity experiments are widely used both for characterization of semiconductor materials and development of new electronic devices. The phenomenon of photoconductivity comprises several processes such as photon absorption, photogeneration and transport of charge carriers, changes in the electronic occupancy of defect energy levels and recombination that are closely related and dependent on the material properties [1]. So, the transient photoconductivity measurements have been proved to be very useful for determination of the charge carriers lifetime as well as for studies of defect centers properties. In particular, a powerful tool for investigations of defects electronic properties in semi-insulating (SI) materials is the high-resolution photoinduced transient spectroscopy (HRPITS) technique [2, 3]. This method involves filling the defects levels with excess charge carriers generated by an optical pulse and advanced analyzing the photocurrent relaxation, observed after switching the light off, as a function of temperature.

The transient photoconductivity has also been studied by computer simulations [4-7]. These investigations are based on the models with assumed properties of defect centers and solutions of the rate equations for photoexcited charge carriers. So far, however, simple models taking into account the two charge states of gold atoms in Si [4], the EL2 and EL3 centers in SI GaAs [5, 6] and two-level schemes for electron and hole trapping in GaN [7] were considered.

The purpose of this paper is to show how the defect centers properties and concentrations in $\mathrm{SI} 4 \mathrm{H}-\mathrm{SiC}$ affect both the rise and the decay of photoconductivity induced by the optical generation of electron-hole-pairs

*corresponding author; e-mail: msuproniuk@wat.edu.pl through band-to-band transitions. The studies are performed by the simulations of photoconductivity kinetics using a model in which six defect centers with various properties are assumed. These are two types of shallow donors, shallow acceptors, deep electron traps, deep hole traps, and recombination centers.

It is worth adding that high-purity, semi-insulating (HPSI) 4H-SiC has recently become an important material for developing advanced photoconductive semiconductor switches (PCSS) for use in high-power and high-temperature applications $[8,9]$. A very high resistivity of this material, greater than $10^{7} \Omega \mathrm{cm}$ at room temperature, is achieved by reducing the concentrations of shallow level impurities, such as residual nitrogen and boron, and introducing native deep-level defects, such as silicon vacancies at various charge states $[2,3]$. However, the properties and concentrations of the deep centers substantially affect the transient characteristics of the PCSS. Acting as traps or recombination centers they have a strong effect on the amplitude, rise time, and fall time of the photocurrent pulses. This has an impact on device performance determining its speed, sensitivity, and noise.

\section{Model}

We have developed a six-level model enabling both the equilibrium conductivity and transient photoconductivity of $4 \mathrm{H}-\mathrm{SiC}$ to be simulated as a function of defect centers properties and concentrations. In this model, two energy levels are related to two types of shallow donors, labeled as SD1 and SD2, which are attributed to nitrogen atoms substituting carbon atoms located in hexagonal $(h)$ and cubic $(k)$ lattice sites, respectively. The other energy levels are related to electron traps (ET), recombination centers (RC), hole traps (HT), and shallow acceptors (SA). For calculations, we have assumed that the properties of electron traps correspond to that of the ID9 centers, which have been widely observed in bulk $4 \mathrm{H}-\mathrm{SiC}$ and were tentatively assigned to native defects [10]. As 
the recombination centers, we have assumed the $Z_{1 / 2}$ centers, which are characteristic of $4 \mathrm{H}-\mathrm{SiC}$ and presumably related to two types of divacancies: the divacancy involving the carbon vacancy in the $h$ site and the silicon vacancy in the $k$ site $\left(\mathrm{V}_{\mathrm{C}}^{h} \mathrm{~V}_{\mathrm{Si}}^{k}\right)$ as well as the divacancy involving the carbon vacancy in the $k$ site and the silicon vacancy in the $h$ site $\left(\mathrm{V}_{\mathrm{C}}^{k} \mathrm{~V}_{\mathrm{Si}}^{h}\right)[10,11]$. As the hole traps properties, we have chosen that of the ID defect centers and the HK3 traps that were studied in $4 \mathrm{H}-\mathrm{SiC}$ by electron paramagnetic resonance (EPR) [12] and deep level transient spectroscopy [13], respectively. The shallow acceptors in our model are the boron atoms substituting the $\mathrm{Si}$ atoms located either in the $h$ or $k$ sites in the $4 \mathrm{H}-\mathrm{SiC}$ lattice [10]. The arrangement in the $4 \mathrm{H}-\mathrm{SiC}$ bandgap at $300 \mathrm{~K}$ of the energy levels assumed for the conductivity simulation is presented in Fig. 1 and the properties of defect centers introducing these levels are listed in Table.

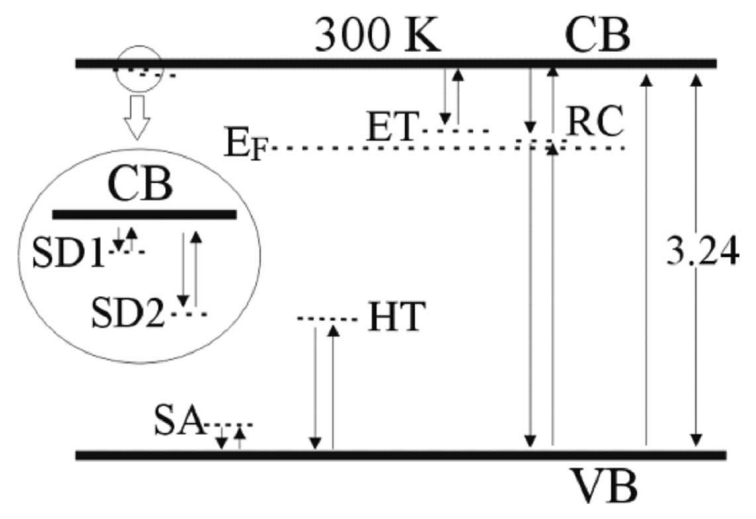

Fig. 1. Schematic energy band diagram for SI 4H-SiC (at $300 \mathrm{~K}$ ) illustrating the defect levels assumed for transient photoconductivity simulation.

Defect centers properties assumed for the conductivity simulation of $4 \mathrm{H}-\mathrm{SiC}$.

TABLE

\begin{tabular}{c|c|c|c|c|c}
\hline \hline \multirow{2}{*}{$\begin{array}{c}\text { Defect } \\
\text { label }\end{array}$} & \multirow{2}{*}{$\begin{array}{c}\text { Defect type } \\
\text { energy }\end{array}$} & \multicolumn{2}{|c|}{$\begin{array}{c}\text { Capture cross-section } \\
{\left[\mathrm{cm}^{2}\right]}\end{array}$} & \multirow{2}{*}{ Identification } \\
\cline { 3 - 5 } & & {$[\mathrm{eV}]$} & Electrons & Holes & \\
\hline SD1 & shallow donor & $E_{\mathrm{c}}-0.050$ & $\approx 4.0 \times 10^{-20}$ & - & $N_{\mathrm{C}}$ in $h$ site [10] \\
SD2 & shallow donor & $E_{\mathrm{c}}-0.092$ & $\approx 4.0 \times 10^{-20}$ & - & $N_{\mathrm{C}}$ in $k$ site [10] \\
ET & electron trap & $E_{\mathrm{c}}-0.555$ & $\approx 1.2 \times 10^{-15}$ & - & ID9 [10] \\
RC & recombination center & $E_{\mathrm{c}}-0.630$ & $1.3 \times 10^{-14}$ & $1.0 \times 10^{-14}$ & $Z_{1 / 2}$ center [10, 11] \\
HT & hole trap & $E_{\mathrm{v}}+1.125$ & - & $\approx 3.1 \times 10^{-14}$ & ID center [12], HK3 trap [13] \\
SA & shallow acceptor & $E_{\mathrm{v}}+0.285$ & - & $\approx 3.1 \times 10^{-17}$ & $\mathrm{~B}_{\mathrm{Si}}$ in both $h$ and $k$ sites [10]
\end{tabular}

The equilibrium material conductivity is defined by the well-known formula:

$$
\sigma_{0}=q\left(n_{0} \mu_{n}+p_{0} \mu_{p}\right) \text {, }
$$

where $q$ is the electronic charge, $n$ and $p$ is the equilibrium electron and hole concentration in the conduction and valence band, respectively, and $\mu_{n}$ and $\mu_{p}$ is the electron and hole mobility, respectively. The equilibrium concentrations of free charge carriers are given by

$$
\begin{aligned}
& \left.n_{0}=N_{\mathrm{C}} \exp \left(E_{\mathrm{F}}-E_{\mathrm{g}}\right) /\left(k_{\mathrm{B}} T\right)\right), \\
& p_{0}=N_{\mathrm{V}} \exp \left(-E_{\mathrm{F}} /\left(k_{\mathrm{B}} T\right)\right),
\end{aligned}
$$

where $N_{\mathrm{C}}$ and $N_{\mathrm{V}}$ denote densities of states in the conduction and valence band, respectively, $E_{\mathrm{g}}$ is the width of the $4 \mathrm{H}-\mathrm{SiC}$ bandgap, $E_{\mathrm{F}}$ is the Fermi level energy, $k_{\mathrm{B}}$ is the Boltzmann constant, and $T$ is the absolute temperature. Taking into account the electron and hole density of states effective masses: $m_{d n}=0.77 m_{0}$ and $m_{d p}=1.0 m_{0}$, respectively, we have $N_{\mathrm{C}}=3.27 \times 10^{15} T^{3 / 2}$ and $N_{\mathrm{V}}=4.83 \times 10^{15} T^{3 / 2} \mathrm{~cm}^{-3}$. The Fermi level position in the bandgap as a function of temperature is calculated by solving the charge neutrality equation

$$
n_{0}-N_{\mathrm{SD} 1}^{+}-N_{\mathrm{SD} 2}^{+}-N_{\mathrm{ET}}^{+}=p_{0}-N_{\mathrm{SA}}^{-}-N_{\mathrm{RC}}^{-}-N_{\mathrm{HT}}^{-},
$$

where $N_{\mathrm{SD} 1}^{+}$and $N_{\mathrm{SD} 2}^{+}$are the concentrations of ionized
SD1 and SD2 shallow donors, $N_{\mathrm{SA}}^{-}$is the concentration of ionized shallow acceptors $\mathrm{SA}, N_{\mathrm{ET}}^{+}$and $N_{\mathrm{HT}}^{-}$are the concentrations of ionized deep electron and hole traps, ET and HT, respectively, and $N_{\mathrm{RC}}^{-}$is the concentration of ionized deep recombination RC. Assuming that $N_{\mathrm{SD}}$, $N_{\mathrm{SA}}, N_{\mathrm{ET}}, N_{\mathrm{HT}}$, and $N_{\mathrm{RC}}$ are the total concentrations of shallow donors, shallow acceptors, electron traps, hole traps and recombination centers, respectively, the concentrations $N_{\mathrm{SD} 1}^{+}, N_{\mathrm{SD} 2}^{+}, N_{\mathrm{SA}}^{-}, N_{\mathrm{ET}}^{+}, N_{\mathrm{HT}}^{-}$, and $N_{\mathrm{RC}}^{-}$can be calculated using the Fermi-Dirac distribution function. The expressions based on this function may be written as

$$
\begin{aligned}
& N_{\mathrm{SD} 1}^{+}=\frac{N_{\mathrm{SD}} / 2}{1+2 \exp \left(\left(E_{\mathrm{F}}-E_{\mathrm{g}}+E_{\mathrm{SD} 1}\right) /\left(k_{\mathrm{B}} T\right)\right)}, \\
& N_{\mathrm{SD} 2}^{+}=\frac{N_{\mathrm{SD}} / 2}{1+2 \exp \left(\left(E_{\mathrm{F}}-E_{\mathrm{g}}+E_{\mathrm{SD} 2}\right) /\left(k_{\mathrm{B}} T\right)\right)}, \\
& N_{\mathrm{SA}}^{-}=\frac{N_{\mathrm{SA}}}{1+\frac{1}{2} \exp \left(\left(E_{\mathrm{SA}}-E_{\mathrm{F}}\right) /\left(k_{\mathrm{B}} T\right)\right)}, \\
& N_{\mathrm{ET}}^{+}=\frac{N_{\mathrm{ET}}}{1+2 \exp \left(\left(E_{\mathrm{F}}-E_{\mathrm{g}}+E_{\mathrm{ET}}\right) /\left(k_{\mathrm{B}} T\right)\right)},
\end{aligned}
$$




$$
\begin{aligned}
& N_{\mathrm{HT}}^{-}=\frac{N_{\mathrm{HT}}}{1+\frac{1}{2} \exp \left(\left(E_{\mathrm{HT}}-E_{\mathrm{F}}\right) /\left(k_{\mathrm{B}} T\right)\right)}, \\
& N_{\mathrm{RC}}^{-}=\frac{N_{\mathrm{RC}}}{1+\frac{1}{2} \exp \left(\left(E_{\mathrm{RC}}-E_{\mathrm{F}}\right) /\left(k_{\mathrm{B}} T\right)\right)},
\end{aligned}
$$

where $E_{\mathrm{SD} 1}, E_{\mathrm{SD} 2}, E_{\mathrm{SA}}, E_{\mathrm{ET}}, E_{\mathrm{HT}}$, and $E_{\mathrm{RC}}$ are the defect centers ionization energies.

According to Eq. (1), the temperature dependence of $\sigma_{0}$ is determined by the relations of $n_{0}$ and $p_{0}$ as a function of temperature as well as through the temperature dependences of the electron and hole mobility. To calculate the Fermi level energy and the values of $n_{0}$ and $p_{0}$ at various temperatures within the range of 200-600 K we have taken into account the temperature changes of the bandgap width given by the following relation [14]:

$$
E_{\mathrm{g}}=3.625-\frac{3.3 \times 10^{-2} T^{2}}{T+1 \times 10^{5}}[\mathrm{eV}] .
$$

The values of $\mu_{n}$ and $\mu_{p}$ at various temperatures within the range of $200-600 \mathrm{~K}$ are calculated using the relations [15]:

$$
\begin{aligned}
& \mu_{n}=\mu_{n}(300 \mathrm{~K}) \times(T / 300)^{\theta_{n}}, \\
& \mu_{p}=\mu_{p}(300 \mathrm{~K}) \times(T / 300)^{\theta_{p}},
\end{aligned}
$$

in which $\mu_{n}(300 \mathrm{~K})$ and $\mu_{p}(300 \mathrm{~K})$ are equal to $950 \mathrm{~cm}^{2} /(\mathrm{V} \mathrm{s})$ and $125 \mathrm{~cm}^{2} /(\mathrm{V} \mathrm{s})$, respectively, $\theta_{n}=$ -2.4 and $\theta_{p}=-2.1$.

The transient photoconductivity induced by the generation of electron-hole pairs by the flux of photons with the energy of $h \nu>E_{\mathrm{g}}$ is given by

$$
\sigma(t)=q\left[n(t) \mu_{n}+p(t) \mu_{p}\right],
$$

where $n(t)$ and $p(t)$ are the concentrations of the excess electrons and holes, respectively. These concentrations may be determined by solving a set of the rate equations taking into account the electronic transitions between the defect levels and the conduction and valence bands

$$
\begin{aligned}
& \frac{\mathrm{d} n(t)}{\mathrm{d} t}=e_{\mathrm{SD} 1} n_{\mathrm{SD} 1}(t)-n(t) c_{\mathrm{SD} 1}\left[N_{\mathrm{SD}} / 2-n_{\mathrm{SD} 1}(t)\right] \\
& +e_{\mathrm{SD} 2} n_{\mathrm{SD} 2}(t)-n(t) c_{\mathrm{SD} 2}\left[N_{\mathrm{SD}} / 2-n_{\mathrm{SD} 2}(t)\right] \\
& -e_{\mathrm{RC}} n_{\mathrm{RC}}(t)+n(t) c_{\mathrm{RC}}\left[N_{\mathrm{RC}}-n_{\mathrm{RC}}(t)\right] \\
& -e_{\mathrm{ET}} n_{\mathrm{ET}}(t)+n(t) c_{\mathrm{ET}}\left[N_{\mathrm{ET}}-n_{\mathrm{ET}}(t)\right] \\
& -\frac{n(t)}{\tau_{n}}+G, \\
& \frac{\mathrm{d} p(t)}{\mathrm{d} t}=e_{\mathrm{RC}}\left[N_{\mathrm{RC}}-n_{\mathrm{RC}}(t)\right]-p(t) c_{\mathrm{RC}} n_{\mathrm{RC}}(t) \\
& +e_{\mathrm{HT}}\left[N_{\mathrm{HT}}-n_{\mathrm{HT}}(t)\right]-p(t) c_{\mathrm{RC}} n_{\mathrm{RC}}(t) \\
& +e_{\mathrm{HT}}\left[N_{\mathrm{HT}}-n_{\mathrm{HT}}(t)\right]-p(t) c_{\mathrm{HT}} n_{\mathrm{HT}}(t) \\
& +e_{\mathrm{SA}}\left[N_{\mathrm{SA}}-n_{\mathrm{SA}}(t)\right]-p(t) c_{\mathrm{SA}} n_{\mathrm{SA}}(t) \\
& -\frac{p(t)}{\tau_{p}}+G
\end{aligned}
$$

$$
\begin{aligned}
& \frac{\mathrm{d} n_{\mathrm{SD} 1}(t)}{\mathrm{d} t}=-e_{\mathrm{SD} 1} n_{\mathrm{SD} 1}(t) \\
& \quad+n(t) c_{\mathrm{SD} 1}\left[N_{\mathrm{SD}} / 2-n_{\mathrm{SD} 1}(t)\right], \\
& \frac{\mathrm{d} n_{\mathrm{SD} 2}(t)}{\mathrm{d} t}=-e_{\mathrm{SD} 2} n_{\mathrm{SD} 2}(t) \\
& \quad+n(t) c_{\mathrm{SD} 2}\left[N_{\mathrm{SD}} / 2-n_{\mathrm{SD} 2}(t)\right], \\
& \frac{\mathrm{d} n_{\mathrm{ET}}(t)}{\mathrm{d} t}=-e_{\mathrm{ET}} n_{\mathrm{ET}}(t)+n(t) c_{\mathrm{ET}}\left[N_{\mathrm{ET}}-n_{\mathrm{ET}}(t)\right], \\
& \frac{\mathrm{d} n_{\mathrm{RC}}(t)}{\mathrm{d} t}=-e_{\mathrm{RC}} n_{\mathrm{RC}}(t)+n(t) c_{\mathrm{RC}}\left[N_{\mathrm{RC}}-n_{\mathrm{RC}}(t)\right] \\
& +e_{\mathrm{RC}}\left[N_{\mathrm{RC}}-n_{\mathrm{RC}}(t)\right]-p(t) c_{\mathrm{RC}} n_{\mathrm{RC}}(t), \\
& \frac{\mathrm{d} n_{\mathrm{HT}}(t)}{\mathrm{d} t}=e_{\mathrm{HT}}\left[N_{\mathrm{HT}}-n_{\mathrm{HT}}(t)\right]-p(t) c_{\mathrm{HT}} n_{\mathrm{HT}}(t),(21) \\
& \frac{\mathrm{d} n_{\mathrm{SA}}(t)}{\mathrm{d} t}=e_{\mathrm{SA}}\left[N_{\mathrm{SA}}-n_{\mathrm{SA}}(t)\right]-p(t) c_{\mathrm{SA}} n_{\mathrm{SA}}(t) .
\end{aligned}
$$

In these equations $G$ denotes the generation rate of electron-hole pairs, $e_{\mathrm{SD} 1}, e_{\mathrm{SD} 2}, e_{\mathrm{ET}}$, and $e_{\mathrm{RC}_{n}}$ denote the thermal emission rates of electrons for the shallow donors of type 1, shallow donors of type 2, electron traps and recombination centers, respectively, and $e_{\mathrm{RC}_{p}}$, $e_{\mathrm{HT}}$, and $e_{\mathrm{SA}}$ are the thermal emission rates of holes for the recombination centers, hole traps, and shallow acceptors, respectively. On the other hand, $c_{\mathrm{SD} 1}, c_{\mathrm{SD} 2}$, $c_{\mathrm{ET}}$ and $c_{\mathrm{RC}_{n}}$ denote the electron capture coefficients for the shallow donors of type 1 , shallow donors of type 2 , electron traps and recombination centers, respectively, and $c_{\mathrm{RC}_{p}}, c_{\mathrm{HT}}$ and $c_{\mathrm{SA}}$ are the hole capture coefficients for the recombination centers, hole traps and shallow acceptors, respectively.

The thermal emission rates are calculated from the well-known Arrhenius equation taking into account the activation energies and capture cross-sections given in Table. In these calculations, the coefficients $\gamma_{n}$ and $\gamma_{p}$ dependent on the effective masses of electrons and holes are equal to $2.5 \times 10^{21}$ and $3.26 \times 10^{21} \mathrm{~cm}^{-2} \mathrm{~s}^{-1} \mathrm{~K}^{-2}$, respectively. The capture coefficients are defined as the products of the capture cross-section and the average thermal velocity of electron or hole. To calculate these coefficients for the assumed defect centers we have used the capture cross-sections given in Table and the relations $v_{n}=7.685 \times 10^{5} T^{0.5}$ and $v_{p}=6.744 \times 10^{5} T^{0.5} \mathrm{~cm} / \mathrm{s}$ for the temperature dependences of average thermal velocities for electron and hole, respectively. The lifetime for electrons or holes is calculated from the formula [1]:

$$
\tau_{n, p}=\frac{1}{N_{\mathrm{CR}} \sigma_{\mathrm{RC} n, p} v_{n, p}} .
$$

The set of rate Eqs. (15)-(22) is numerically solved by means of the calculation procedures using the RungeKutta-Fehlberg method.

\section{Simulations results}

Simulations of both the equilibrium conductivity and transient photoconductivity of $\mathrm{SI} 4 \mathrm{H}-\mathrm{SiC}$ were performed using MATLAB software tools enabling the nonlinear 
and rate equations to be solved. The equilibrium electron concentrations on the defects levels were used as the starting conditions for solving the rate equations. The simulated changes of the material equilibrium conductivity as a function of temperature for the assumed concentrations of SD, SA, ET, RC, and $\mathrm{HT}$ that are equal to $1 \times 10^{16}, 9.7 \times 10^{15}, 5 \times 10^{14}, 8 \times 10^{14}$ and $7 \times 10^{14} \mathrm{~cm}^{-3}$, respectively, are presented in Fig. 2. The values of conductivity at temperatures 300,350 , and $400 \mathrm{~K}$ shown in Fig. 2 correspond to the values of resistivity of $2.17 \times 10^{8}$, $7.80 \times 10^{6}$, and $6.62 \times 10^{5} \Omega \mathrm{cm}$, respectively, and the Fermi level positions with respect of the conduction band minimum $\left(E_{\mathrm{c}}-E_{\mathrm{F}}\right)$ are $0.695,0.715$, and $0.720 \mathrm{eV}$, respectively. In other words, for the given concentrations of defect centers, the material is semi-insulating and the Fermi level shifts towards the midgap with increase of the temperature. The decrease in the resistivity is due to increase of the free electron concentration in the conduction band that at temperatures 300,350 , and $400 \mathrm{~K}$ is equal to $3.03 \times 10^{7}, 1.22 \times 10^{9}$ and $1.98 \times 10^{10} \mathrm{~cm}^{-3}$, respectively. The rise in the electron concentration in the conduction band results from the decrease in the electronic occupancy of the $Z_{1 / 2}$ recombination centers.

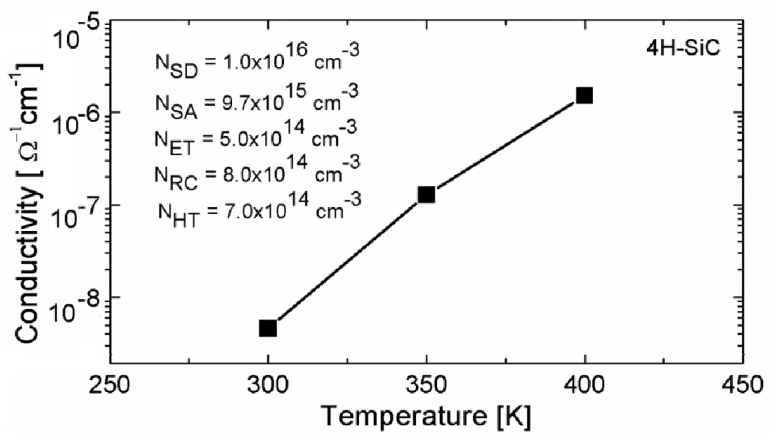

Fig. 2. Simulated temperature dependence of SI $4 \mathrm{H}-\mathrm{SiC}$ dark conductivity for the properties and concentrations of defect centers listed in Table.

The effect of temperature on transient photoconductivity of $\mathrm{SI} 4 \mathrm{H}-\mathrm{SiC}$ is presented in Fig. 3. The curves show the evolution of the material photoconductivity in time after switching on at $t=0$ the band-to-band generation of electron-hole pairs with a rate $G=3.31 \times 10^{22} \mathrm{~cm}^{-3} / \mathrm{s}$ as well as after switching off the generation excess charge carriers at $t=10 \mu \mathrm{s}$. The photoconductivity decay after termination of the generation lasts $10 \mu \mathrm{s}$, so the duration time of the whole transients is $20 \mu \mathrm{s}$. The simulation results indicate that both the rise and decay of photoconductivity are dependent on the temperature. The rise curves can be divided into three parts: the first part lasting up to $\approx 1 \times 10^{-8} \mathrm{~s}$, the second part lasting from $\approx 1 \times 10^{-8} \mathrm{~s}$ to $\approx 1 \times 10^{-7} \mathrm{~s}$, and the third, almost steady state part, lasting from $\approx 2 \times 10^{-7} \mathrm{~s}$ to $\approx 1 \times 10^{-5} \mathrm{~s}$. The first part is controlled by the lifetimes of electrons and holes that at $300 \mathrm{~K}$ are $\approx 7.2 \times 10^{-9} \mathrm{~s}$ to $\approx 1.1 \times 10^{-8} \mathrm{~s}$, respectively, and slightly decrease with increase of the temperature. The second part is also influenced by the capture of charge carriers on the deep levels of electron traps, hole traps and recombination centers. The electron capture coefficients for the electron traps and recombination centers at $300 \mathrm{~K}$ are $\approx 1.6 \times 10^{-8}$ and $1.7 \times 10^{-7} \mathrm{~cm}^{3} / \mathrm{s}$. The hole capture coefficients for the hole traps and recombination centers at $300 \mathrm{~K}$ are $\approx 3.6 \times 10^{-7}$ and $\approx 1.2 \times 10^{-7} \mathrm{~cm}^{3} / \mathrm{s}$, respectively. It is worth adding that the capture coefficients slightly increase with increase of the temperature. The third part of the photoconductivity rise is affected both by capture and thermal emission of charge carriers from the defect levels. The most influential is the thermal emission of electrons from the shallow donors and the thermal emission of holes from the shallow acceptors. For the shallow donors of SD1 and SD2, the electron emission rates at the temperatures of 300,350 , and $400 \mathrm{~K}$ are: $1.3 \times 10^{6}$ and $2.6 \times 10^{6} \mathrm{~s}^{-1}, 2.3 \times 10^{6}$ and $5.8 \times 10^{6} \mathrm{~s}^{-1}$, and $3.74 \times 10^{6}$ and $1.1 \times 10^{7} \mathrm{~s}^{-1}$, respectively. For the shallow acceptors, the hole emission rates at these temperatures are $1.5 \times 10^{5}, 9.7 \times 10^{5}$, and $4.1 \times 10^{6} \mathrm{~s}^{-1}$, respectively.

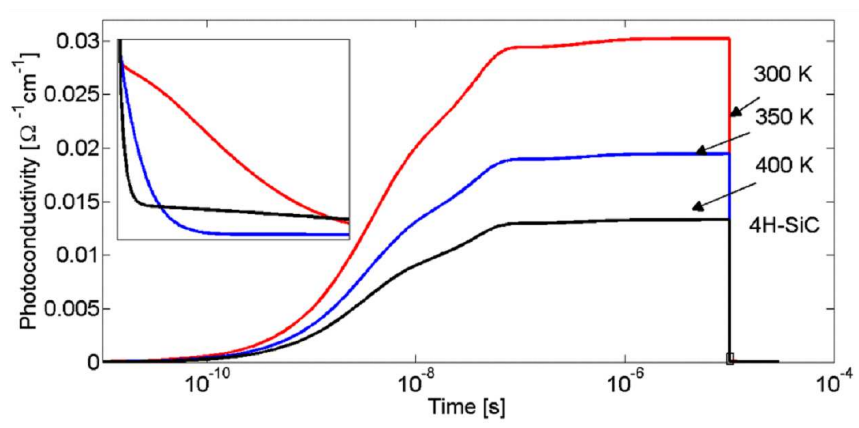

Fig. 3. Effect of temperature on transient photoconductivity of SI $4 \mathrm{H}-\mathrm{SiC}$ for the properties and concentrations of defect centers listed in Table. The generation rate of electron-hole pairs is $3.31 \times 10^{22} \mathrm{~cm}^{-3} / \mathrm{s}$. The inset shows the relaxation after switching off the generation of excess charge carriers.

At around $9 \mu \mathrm{s}$ (Fig. 3), just before switching off the generation of electron-hole pairs, the photoconductivity reaches a steady state. At the generation rate of $3.31 \times 10^{22} \mathrm{~cm}^{-3} / \mathrm{s}$, the steady-state electron and hole concentrations at $300 \mathrm{~K}$ are $1.66 \times 10^{14}$, and $2.45 \times$ $10^{14} \mathrm{~cm}^{-3}$, respectively, and for these concentrations the quasi-Fermi levels for electrons and holes are located at around $E_{\mathrm{c}}-0.30 \mathrm{eV}$ and $E_{\mathrm{v}}+0.30$, respectively. The higher concentration of excess holes than that of excess electrons results from the longer lifetime of holes $\left(\tau_{p}=1.1 \times 10^{-8} \mathrm{~s}\right)$ compared to that of electrons $\left(\tau_{n}=7.2 \times 10^{-9} \mathrm{~s}\right)$. According to the model, the electron and hole lifetimes slightly decrease with temperature, for their temperature changes (Eq. (23)) are controlled by the temperature dependences of the average thermal velocities. Thus, the steady-state electron and hole concentrations also slightly decrease with temperature and at $400 \mathrm{~K}$ they are $1.44 \times 10^{14}$ and $2.13 \times 10^{14} \mathrm{~cm}^{-3}$, 
respectively. Therefore, the significant decrease in the steady-state photoconductivity with temperature seen in Fig. 3 results from substantial diminishing of the electron and hole mobilities with increasing temperature. According to the model, these mobilities at the temperatures of 300,350 , and $400 \mathrm{~K}$ are 950 and $125 \mathrm{~cm}^{2} /(\mathrm{V} \mathrm{s}), 656$ and $90 \mathrm{~cm}^{2} /(\mathrm{Vs})$, and 476 and $68 \mathrm{~cm}^{2} /(\mathrm{V} \mathrm{s})$.

The photoconductivity relaxation waveforms after switching off the generation of electron-hole pairs (Fig. 3) consist of two parts. The first dominating part is very fast and is due to the recombination of excess electrons and holes controlled by their lifetimes. The second part, which is shown in the inset, is much slower and related to the thermal emission of electrons from the electron traps and recombination centers. The effect of temperature on the decay rate of the waveforms presented in the inset is clearly material seen.

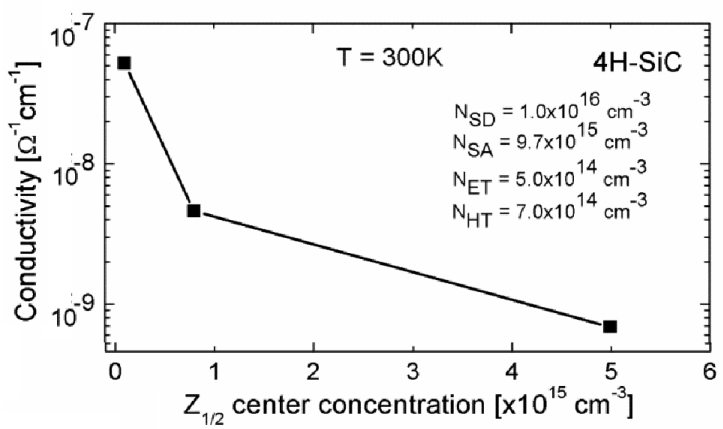

Fig. 4. Simulated dark conductivity of SI $4 \mathrm{H}-\mathrm{SiC}$ versus the $Z_{1 / 2}$ center concentration.

In Fig. 4 we demonstrate the effect of the $Z_{1 / 2}$ center concentration on the equilibrium conductivity of $4 \mathrm{H}-\mathrm{SiC}$. The substantial decrease in the conductivity with increase of the $Z_{1 / 2}$ center concentration from $1 \times 10^{14}$ to $5.0 \times 10^{15} \mathrm{~cm}^{-3}$ is observed. In other words the material becomes more semi-insulating as a result of the Fermi level shift towards the middle of the bandgap from 0.635 to $0.745 \mathrm{eV}$ with respect to the conduction band minimum. The rise in the material resistivity corresponding to this shift is from $1.9 \times 10^{7}$ to $1.5 \times 10^{9} \Omega \mathrm{cm}$.

The effect of the $Z_{1 / 2}$ center concentration on the transient photoconductivity of SI $4 \mathrm{H}-\mathrm{SiC}$ at $300 \mathrm{~K}$ is demonstrated in Fig. 5. It is seen that the increase in the $Z_{1 / 2}$ center concentration results in the substantial drop of the steady state photoconductivity. This effect was expected as in our model the $Z_{1 / 2}$ center is the recombination center and its concentration directly determines the electron and hole lifetimes and the steady state concentrations of the excess charge carriers. For the $Z_{1 / 2}$ center concentrations given in Fig. 5, namely $1.0 \times 10^{14}$, $8.0 \times 10^{14}$, and $5.0 \times 10^{15} \mathrm{~cm}^{-3}$, the electron lifetimes are $5.8 \times 10^{-8}, 7.2 \times 10^{-9}$, and $1.1 \times 10^{-9} \mathrm{~s}$, respectively, and the hole lifetimes are equal to $8.6 \times 10^{-8}, 1.1 \times 10^{-8}$, and $1.7 \times 10^{-9} \mathrm{~s}$, respectively. The steady state excess electron concentrations are $1.6 \times 10^{15}, 1.7 \times 10^{14}$, and

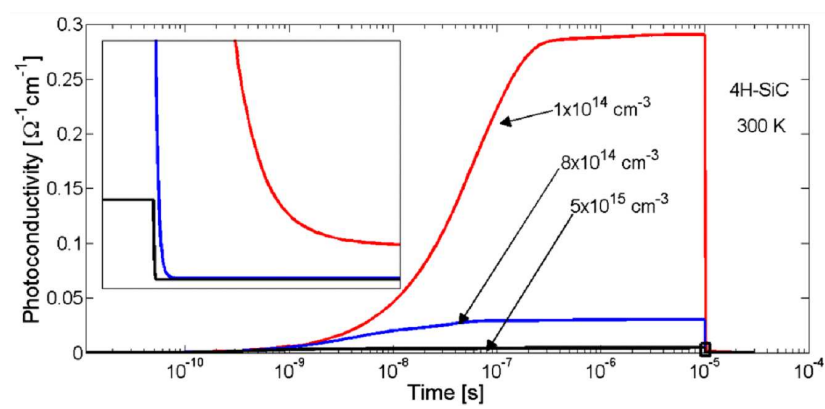

Fig. 5. Effect of the $Z_{1 / 2}$ center concentration on transient photoconductivity of SI $4 \mathrm{H}-\mathrm{SiC}$. The generation rate of electron-hole pairs is $3.31 \times 10^{22} \mathrm{~cm}^{-3} / \mathrm{s}$. The inset shows the relaxation after switching off the generation of excess charge carriers.

$2.6 \times 10^{13} \mathrm{~cm}^{-3}$, respectively, and the excess hole concentrations are $2.4 \times 10^{15}, 2.5 \times 10^{14}$, and $3.8 \times 10^{13} \mathrm{~cm}^{-3}$, respectively. The inset in Fig. 5 shows the end parts of the relaxation waveforms after switching of the generation of electron-hole pairs. At the low $Z_{1 / 2}$ center concentration the thermal emission from defect centers is very well seen. At the middle concentration of this center we can observe only the fastest part of the relaxation waveform related to thermal emission and at the high $Z_{1 / 2}$ center concentration the waveform related to thermal emission is not resolved in the photoconductivity decay.

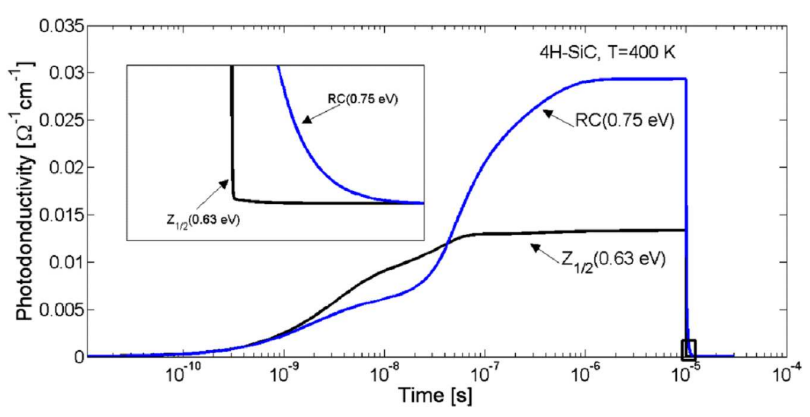

Fig. 6. Comparison of simulated transient photoconductivity at $400 \mathrm{~K}$ for $\mathrm{SI} 4 \mathrm{H}-\mathrm{SiC}$ with different properties of recombination centers. The generation rate of electron-hole pairs is $3.31 \times 10^{22} \mathrm{~cm}^{-3} / \mathrm{s}$. The inset shows the relaxation after switching off the generation of excess charge carriers.

Figure 6 illustrates the $4 \mathrm{H}-\mathrm{SiC}$ photoconductivity time evolution in the case of recombination centers with different properties. It is seen that the $Z_{1 / 2}$ center is significantly more efficient recombination center than an acceptor center with the activation energy of $E_{\mathrm{c}}-0.75 \mathrm{eV}$, electron capture cross-section of $2.4 \times 10^{-14} \mathrm{~cm}^{2}$ and hole capture cross-section of $1.0 \times 10^{-15} \mathrm{~cm}^{2}$. Compared to the $Z_{1 / 2}$ center parameters given in Table, the $0.75 \mathrm{eV}$ recombination center is characterized by the deeper activation energy for thermal emission of electrons, approximately twice higher electron capture cross-section and by 
the order of magnitude lower hole capture cross-section. The concentrations of the $Z_{1 / 2}$ center and the $0.75 \mathrm{eV}$ center are the same and equal to $8.0 \times 10^{14} \mathrm{~cm}^{-3}$. In view of recent results, the $Z_{1 / 2}$ center can be removed by a heat treatment at the temperature $\approx 1600^{\circ} \mathrm{C}[16]$. It is very likely that the annihilation of the $Z_{1 / 2}$ center is accompanied by the deformation of the $0.75 \mathrm{eV}$ center $[2,16,17]$.

For the material with the $0.75 \mathrm{eV}$ recombination center the lifetimes of excess electrons and holes are equal to $3.9 \times 10^{-9}$ and $1.1 \times 10^{-7} \mathrm{~s}$. These data indicate that the hole lifetime is by more than one order of magnitude longer compared to the electron lifetime. So, the rise part of the photoconductivity transient for the $4 \mathrm{H}-\mathrm{SiC}$ with the $0.75 \mathrm{eV}$ recombination center (Fig. 6) depicts the changes with time of the hole concentration. At the steady state, the concentrations of excess electrons and holes are $9.0 \times 10^{13}$ and $2.5 \times 10^{15} \mathrm{~cm}^{-3}$, respectively. The inset in Fig. 6 illustrates different relaxation waveforms for the material with the $Z_{1 / 2}$ center and that with the $0.75 \mathrm{eV}$ recombination center. It is clearly seen that the photoconductivity relaxation related to the thermal emission of charge carriers from defect levels for the material with the $0.75 \mathrm{eV}$ recombination center is strongly enhanced.

\section{Experimental results}

The simulated photoconductivity transients have been compared with experimental photocurrent transients recorded at $400 \mathrm{~K}$ for a sample of high purity, semi-insulating $4 \mathrm{H}-\mathrm{SiC}$ before and after annealing at $\approx 1670{ }^{\circ} \mathrm{C}$.

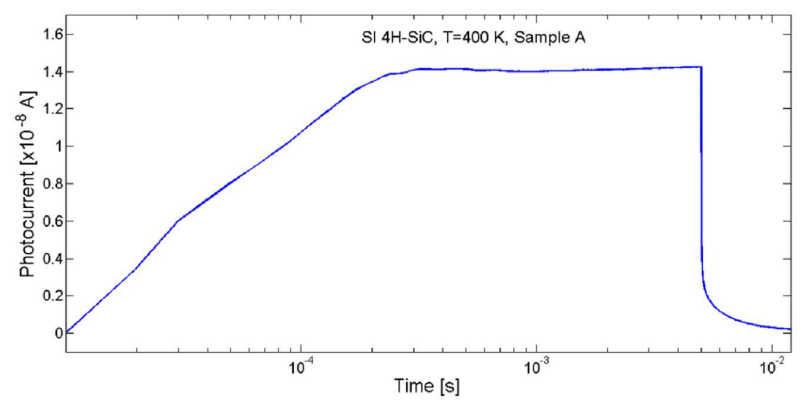

Fig. 7. Experimental photocurrent transient at $400 \mathrm{~K}$ for a sample of SI $4 \mathrm{H}-\mathrm{SiC}$ not subjected to heat treatment. The $Z_{1 / 2}$ center concentration in the sample is $\approx 7 \times 10^{14} \mathrm{~cm}^{-3}$

These photocurrent transients are shown in Fig. 7 and Fig. 8, respectively. The heat treatment of the sample with a resistivity of $\approx 10^{8} \Omega \mathrm{cm}$ was carried out in an epitaxial reactor for $1 \mathrm{~h}$. The measurements of photocurrent transients were performed using an experimental system dedicated to studies of defect levels by the HRPITS method [2]. The transient were amplified by means of a Keithley 428 fast picoammeter and then digitized with a

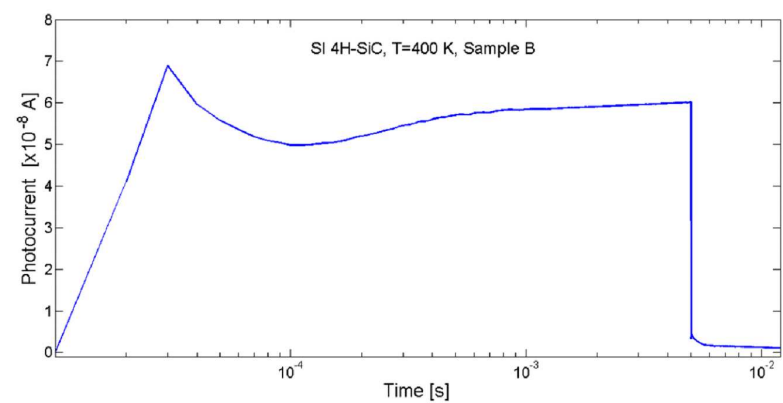

Fig. 8. Experimental photocurrent transient at $400 \mathrm{~K}$ for a sample of $\mathrm{SI} 4 \mathrm{H}-\mathrm{SiC}$ after heat treatment at $\approx 1670^{\circ} \mathrm{C}$. Instead of the $Z_{1 / 2}$ center, centers with the activation energies of $750 \mathrm{meV}$ and $830 \mathrm{meV}$ are present in this sample. The concentrations of these centers are $\approx 5 \times 10^{13} \mathrm{~cm}^{-3}$ and $\approx 3 \times 10^{14} \mathrm{~cm}^{-3}$, respectively.

12-bit amplitude resolution and a $1 \mu$ s time resolution. To improve the signal to noise ratio, the digital data were averaged taking 500 waveforms. The excess charge carriers were generated in the region between two coplanar $\mathrm{Au} / \mathrm{Cr}$ ohmic contacts by UV pulses with the wavelength of $375 \mathrm{~nm}$ (photon energy $3.31 \mathrm{eV}$ ) emitted by a semiconductor laser. The length of excitation pulses was $5 \mathrm{~ms}$ and the repetition period was $500 \mathrm{~ms}$. The photon flux, controlled by the laser bias, was $\approx 2.8 \times 10^{17} \mathrm{~cm}^{-2} \mathrm{~s}^{-1}$. The voltage between two coplanar contacts was $20 \mathrm{~V}$.

The photocurrent transient measured for the non annealed sample of SI $4 \mathrm{H}-\mathrm{SiC}$ (Fig. 7) is mainly affected by the $Z_{1 / 2}$ center detected in this sample by the HRPITS technique. The activation energy of this center is $\approx 630 \mathrm{meV}$ and its concentration is $\approx 7 \times 10^{14} \mathrm{~cm}^{-3}$. Although we have different values on the time axis, the shape of the waveform in Fig. 7 is very similar to the simulated waveform shown in Fig. 6 that was calculated at the assumption that the $Z_{1 / 2}$ center acts as a recombination center and its concentration is $8 \times 10^{14} \mathrm{~cm}^{-3}$. It is worth adding that in the non annealed material an electron trap with the activation energy of $560 \mathrm{meV}$ corresponding to the ET trap assumed for the simulation has also been revealed and its concentration was found to be $\approx 1 \times 10^{14} \mathrm{~cm}^{-3}$.

After the annealing, the $Z_{1 / 2}$ center disappeared. So, the photocurrent transient shown in Fig. 8 is influenced by other deep defect centers that were formed as a result of defect reactions during the heat treatment. According to the HRPITS results, the activation energies of these centers are $750 \mathrm{meV}$ and $830 \mathrm{meV}$. Similarly as in Fig. 6, the shape of this transient is different than that for the material containing the $Z_{1 / 2}$ center. In particular, the height of the photocurrent pulse at the moment of switching off the optical excitation is several times lower in the case of the presence of the $Z_{1 / 2}$ center (Fig. 7 ) than that in the case of presence of the $750 \mathrm{meV}$ and $830 \mathrm{meV}$ defect centers (Fig. 8). The same feature is observed from the simulated transient photoconductivity results presented in Fig. 6. Thus, both the simulated and experimental 
results indicate that the $Z_{1 / 2}$ center is the main defect center in $4 \mathrm{H}-\mathrm{SiC}$ that has a detrimental effect for the material transient photoconductivity.

\section{Conclusions}

A model enabling the equilibrium conductivity and transient photoconductivity of $\mathrm{SI} 4 \mathrm{H}-\mathrm{SiC}$ to be simulated has been demonstrated. The model is based on solving the charge neutrality equation and a set of differential rate equations for the assumed parameters and concentrations of defect centers such as shallow donors, shallow acceptors, deep electron traps, deep hole traps, and recombination centers. Using this model, the simulations of both equilibrium conductivity and transient photoconductivity have been carried out. It is shown that the evolution of photoconductivity in time after switching on the band-to-band generation of electron-hole pairs is strongly affected by the recombination rate of the excess electrons and holes, as well as by the rate of capture of excess charge carriers from the conduction and valence band by deep level defects and the thermal emission of the carriers to the bands from defect levels. The results of transient photoconductivity simulations have been compared with the experimental photocurrent transients for a sample of high purity, semi-insulating $4 \mathrm{H}-\mathrm{SiC}$ before and after annealing at $\approx 1670{ }^{\circ} \mathrm{C}$. The results of transient photocurrent measurements confirm the simulations results indicating that the $Z_{1 / 2}$ center is a very effective recombination center in $\mathrm{SI} 4 \mathrm{H}-\mathrm{SiC}$ having detrimental effect on the transient photoconductivity.

\section{References}

[1] R.H. Bube, Photoelectric Properties of Semiconductors, Cambridge University Press, Cambridge 1992.

[2] P. Kamiński, R. Kozłowski, M. Miczuga, M. Pawłowski, M. Kozubal, M.A. Pawłowski, J. Mater. Sci., Mater. Electron. 19, 224 (2008).
[3] M.V.S. Chandrashekhar, I. Choudhury, P. Kaminski, R. Kozlowski, P.B. Klein, T. Sudarshan, Appl. Phys. Expr. 5, 025502 (2012).

[4] H. Kimura, T. Kurosu, Y. Akiba, M. Iida, Jpn. J. Appl. Phys. 32, 741 (1993).

[5] T. Hahn, S. Schmerler, S. Hahn, J.R. Niklas, B. Gruendig-Wendrock, J. Mater. Sci., Mater. Electron. 19, 79 (2008).

[6] B. Berger, N. Schüler, S. Anger, B. GründigWendrock, J.R. Niklas, K. Dornich, Phys. Status Solidi A 4, 769 (2011).

[7] M. Salis, A. Anedda, F. Quarati, J. Blue, W. Cunningham, J. Appl. Phys. 97, 033709 (2005).

[8] S. Doğan, A. Teke, D. Huang, H. Morkoç, C.B. Roberts, J. Parish, B. Ganguly, M. Smith, R.E. Myers, S.E. Saddow, Appl. Phys. Lett. 82, 3107 (2003).

[9] C. Hettler, W.W. Sullivan III, J. Dickens, Mater. Sci. Forum 717-720, 301 (2012).

[10] M. Kozubal, Ph.D. Thesis, Institute of Electronic Materials Technology, Warszawa 2011.

[11] P. Kaminski, M. Kozubal, J.D. Caldwell, K.K. Kew, B.L. VanMil, R.L. Myers-Ward, C.R. Eddy Jr., D.K. Gaskill, Electronic Materials ITME 38, 26 (2010).

[12] M.E. Zvanut, V.V. Konovalov, Appl. Phys. Lett. 80, 410 (2002).

[13] K. Danno, T. Kimoto, Mater. Sci. Forum 527-529, 501 (2006).

[14] M. Lades, Ph.D. Thesis, Munich Technical University München 2000.

[15] S. Kagamihara, H. Matsuura, T. Hatakeyama, T. Watanabe, M. Kushibe, T. Shinohe, K. Arai, J. Appl. Phys. 96, 5601 (2004).

[16] L. Storasta, H. Tsuchida, Appl. Phys. Lett. 90 062116 (2007).

[17] L.S. Lovlie, B.G. Svensson, Phys. Rev. B 86, 075205 (2012). 